\title{
Jump Drive, Replicators, Transporters, and Time Travel
}

\author{
Yosef Joseph Segman* \\ Independent Researcher, Zichron Ya'acov, Israel \\ Email: yosef@void2life.com
}

How to cite this paper: Segman, Y.J. (2021) Jump Drive, Replicators, Transporters, and Time Travel. Advances in Aerospace Science and Technology, 6, 123-133.

https://doi.org/10.4236/aast.2021.63009

Received: June 11, 2021

Accepted: July 12, 2021

Published: July 15, 2021

Copyright (c) 2021 by author(s) and Scientific Research Publishing Inc. This work is licensed under the Creative Commons Attribution-NonCommercial International License (CC BY-NC 4.0). http://creativecommons.org/licenses/by-nc/4.0/ (c) (i) $\$$ Open Access

\begin{abstract}
Jumping from place to place, replicating food, biological or mechanical parts or beaming up somebody, may not be fiction, rather an issue of practical implementation as shall be observed in this paper. Devices like transporter, food replicators or warp drive intrigue our imagination. This paper is intended to show that Jump drive is an issue of coordinate transformation. Changing location from planet $\mathrm{X}$ to planet $\mathrm{Y}$ does not necessarily require travelling a distance $\mathrm{D}$ connecting between the two planets. The theoretical knowledge of changing the location from coordinate $\mathrm{X}$ to coordinate $\mathrm{Y}$ exists; we do that in signal processing, but, we have not yet developed such a machine. The present paper shows the feasibility of jump drive; however, much work needs to be done on the implementation.
\end{abstract}

\section{Keywords}

Fourier Transform, Spatial Shift, Time Shift, Coordinates Transformation, Time Travel, Discontinuous Travel, Transporters, Replicators, Causality, Noncausality

\section{Introduction}

The idea of space traveling intrigues humanity to wander in space beyond our solar system, within and beyond the milky way galaxy. Traveling long distance has a major challenge-speed. Physics teaches us that speed is bounded by the speed of light. Furthermore, even though we can travel at speed of light and above we still need to go through all the points connecting between planet $\mathrm{A}$ and planet $\mathrm{B}$. This assumption may not be adequate.

Matter is a logical perception, even though we are experiencing the law of *https://www.void2life.com, Void2life is a voluntary organization sharing knowledge and wisdom to promote humanity to its next level of consciousness evolution. 
physics; eventually all the scenes we are experiencing are cords of consciousness (Y.J. Segman [1]) and that includes the existing of matter out of total void, (Y. J. Segman [2]). The sensation of reality depends on our consciousness flexibility absorbing reality as stiff or as one that can be shaped in a way that the Universe coordinate system can be manipulated.

M. Alcubierre [3] suggested warp drive overcoming light years distance between two potential stars. Yet, it still requires travelling in space between the points connecting the two stars. Furthermore, Alcubierre model produces negative energy everywhere. Alternative geometries were proposed by Y. J. Segman [4] resulting in positive warp drive energy.

This paper introduces additional concept to warp drive, the jump drive i.e. discontinuous travel, where we show that there is no need to travel the points connecting the two planets. The idea is based on the shifting property of the Fourier transform. The main practical issue is the implementation of the Fourier transform on real objects. Based on the same idea, we may develop replicators and transporters handheld devices.

Section 2 provides preliminaries. Section 3 discusses the suggested method where the object assumed to be fixed during the execution of the Fourier transform. Section 4 shortly discusses the way back from planet A. The suggested method may also be used for parts and food replicators as shortly discussed in Section 5. Time and potential time travel are being discussed in Section 6, i.e. time travel is a time shift of the current timeline. Since the Fourier transform is a non-causal operator, we may assume that there is no guarantee to keep the same past events before the execution of the Fourier transform or the future events to be occurred after the execution of the Fourier transform. Section 7 further discusses causality and non-causality which may affect the appearance of the object in the current space-time universe. Section 8 suggests potential implementation of holographic messages and Section 9 discusses some of the challenges of the practical implementation.

\section{Preliminaries}

Let $X=(x, y, z)$ be a universal flat spatial coordinate system describing the universal location. It can be geographical location or functional location depending on the local forces (i.e., gravitation, anti-gravitation, pressure, heat, etc.) that may influence the universal coordinate location $X$.

For simplicity we just use Geographical Universal coordinate location, where $(0,0,0)$ represents the Universe (Cosmos) center. Earth would be at the Universal location $X^{0}$ and a planet A for example at the location $X^{1}$. For the purpose of this paper, we assume universal time since of its initialization e.g. big bang. For example, $\left(X^{0}, t_{0}\right)$ and $\left(X^{1}, t_{0}\right)$ means the coordinate locations of Earth and of planet $A$ at time $t_{0}$ since of the moment of the universe initialization.

Let $D$ be the distance vector connecting between Earth to a planet A. Travelling at speed of light a distance $D=\left(d_{x}, d_{y}, d_{z}\right)$ from Earth to a place located 
on planet at $X^{1}=\left(x_{1}, y_{1}, z_{1}\right)=\left(x_{0}+d_{x}, y_{0}+d_{y}, z_{0}+d_{z}\right)$ may require few dozen light years. For example, if $\mathrm{A}$ is a planet nearby Arcturus, then the journey at speed of light may take over 35 years Earth time.

Our aim is to jump from $U\left(X^{0}\right)$ at certain local time on planet Earth into $U\left(X^{0}+D\right)=U\left(X^{1}\right)$ in plant A. $U$ stands for the universe i.e. Cosmos.

Let,

$$
\mathcal{F}(\Omega)=c \int_{R^{3}} f(X) \mathrm{e}^{-2 \pi i \Omega X} \mathrm{~d} X
$$

be the 3D spatial Fourier transform of $f(X), c>0$ is a constant.

The inverse Fourier transform is,

$$
f(X)=c \int_{R^{3}} \mathcal{F}(\Omega) \mathrm{e}^{2 \pi i \Omega X} \mathrm{~d} \Omega
$$

A basic relationship of the Fourier transform under shift operation is,

$$
\mathcal{F}_{D}(\Omega)=c \int_{R^{3}} f(X+D) \mathrm{e}^{-2 \pi i \Omega X} \mathrm{~d} X=\mathrm{e}^{2 \pi i \Omega D} \mathcal{F}(\Omega)
$$

where $f(X)$ admits the requirement for Fourier transform. In fact, all equivalent groups to the additive group will admit the shifting property of the Fourier Transform. A proof and a flow chart how to compute the new coordinate system and the associated Fourier kernel is provided in Y. J. Segman et al. [5]-[11].

Let $\mathrm{J}_{\mathrm{E}}$ be a transporter device with $S_{E}^{2}$ sphere geometry located on planet Earth at the coordinate $X^{0}=\left(x_{0}, y_{0}, z_{0}\right)$ where,

$$
S_{E}^{2}=\left\{X: r \leq 1 ; r^{2}=\left(x-x_{0}\right)^{2}+\left(y-y_{0}\right)^{2}+\left(z-z_{0}\right)^{2}, \text { otherwise } 0\right\}
$$

$S_{E}^{2}$ represents the inside of the device $\mathrm{J}_{\mathrm{E}}$. Sphere geometry is not unique. It is convenience for the purpose of this paper. In the future we may develop personal handheld device.

\section{The Concept}

Let $f\left(X^{0}\right)$ be the object located within the device $J_{E}$ on planet Earth. The goal is to transfer $f\left(X^{0}\right)$ from the device $\mathrm{J}_{\mathrm{E}}$ into $f\left(X^{0}+D\right)=f\left(X^{1}\right)$ which is now being located on planet $A$.

Time is the local time of the event on planet Earth. We assume that the object $f(X)$ remains "as is" during the time of the execution of the Fourier transform.

The steps are given theoretically, of course it requires a real design of the approach, but the idea is that it is feasible to jump from planet Earth to plant A independent of the distance connecting between them, in fact, a similar version can be used to beam up somebody or replicate food, biological or mechanical parts.

The first step is to execute Fourier transform over all spherical points within the device $\mathrm{J}_{\mathrm{E}}$. In case $f(X)$ represents the object within the device $\mathrm{J}_{\mathrm{E}}$, then $f(X)$ is zero outside itself (i.e. $f(X)$ is the object to be transformed to planet $\mathrm{A}$ ) and that includes the outside the device $\mathrm{J}_{\mathrm{E}}$. For simplicity we consider $f(X)$ to represent the object and the internal surrounding space within $\mathrm{J}_{\mathrm{E}}$. The material within the transporter $\mathrm{J}_{\mathrm{E}}$ may be air, water or other material that may support the process. 
The outcome is $\mathcal{F}(\Omega)$ i.e.

$$
\mathcal{F}(\Omega)=c \int_{R^{3}} f(X) \mathrm{e}^{-2 \pi i \Omega X} \mathrm{~d} X=c \int_{S_{E}^{2}} f(X) \mathrm{e}^{-2 \pi i \Omega X} \mathrm{~d} X
$$

where $\Omega X=\Omega_{x} x+\Omega_{y} y+\Omega_{z} z$.

Second step is multiplying $\mathcal{F}(\Omega)$ by $\mathrm{e}^{2 \pi i \Omega D}$ i.e. (Equation (3)),

$$
\mathcal{F}(\Omega) \mathrm{e}^{2 \pi i \Omega D}=\mathcal{F}_{D}(\Omega)
$$

where $D$ is a directional distance vector connecting planet Erath to planet A, $\Omega D=\Omega_{x} d_{x}+\Omega_{y} d_{y}+\Omega_{z} d_{z}$.

Equation (6) is the trickiest part. Since it does not depend on the actual location of the transporter $\mathrm{J}_{\mathrm{E}}$. This means that the integration over the sphere $S_{E}^{2}$ remains unchanged even though there was a change in the integration variables. Elaborating this issue, let for example consider the square impulse function,

$$
I(x)= \begin{cases}1 & -1 \leq x \leq 1 \\ 0 & \text { otherwise }\end{cases}
$$

The Fourier transform of $I(x)$

$$
c \int_{-\infty}^{+\infty} I(x) \mathrm{e}^{-2 \pi i \omega x} \mathrm{~d} x=c \int_{-1}^{+1} I(x) \mathrm{e}^{-2 \pi i \omega x} \mathrm{~d} x=\hat{I}(\omega)
$$

Shifting $I(x)$ by $d$

$$
I(x+d)=\left\{\begin{array}{cc}
1 & -1 \leq x+d \leq 1 \\
0 & \text { otherwise }
\end{array}\right.
$$

means that $x+d$ is no longer in the original interval $[-1,1]$ since

$$
I(x+d)=\left\{\begin{array}{cc}
1 & -1-d \leq x \leq 1-d \\
0 & \text { otherwise }
\end{array}\right.
$$

Yet, since the Fourier transform is a non-causal transformation which means that the integration remains unchanged under shifting since the shifting element $d$ has been changed from spatial shift element into phase element. Therefore, the Fourier transform of Equations (8), (9) shall remain in the interval $[-1,1]$ after changing the integration variable, i.e.

$$
c \int_{-\infty}^{+\infty} I(x+d) \mathrm{e}^{-2 \pi i \omega x} \mathrm{~d} x=c \int_{-\infty}^{+\infty} I(u) \mathrm{e}^{-2 \pi i \omega(u-d)} \mathrm{d} u=\mathrm{e}^{2 \pi i \omega d} \hat{I}(\omega)
$$

This means that the integration within the device $\mathrm{J}_{\mathrm{E}}$ of the forward-backward Fourier transforms Equations (2), (5) and (6) remains on planet Earth.

Next step is the execution of the inverse Fourier transform as defined in Equation (2),

$$
f\left(X^{0}+D\right)=f\left(X^{1}\right)=c \int_{R^{3}} \mathcal{F}_{D}(\Omega) \mathrm{e}^{2 \pi i \Omega X^{0}} \mathrm{~d} \Omega=c \int_{R^{3}} \mathcal{F}(\Omega) \mathrm{e}^{2 \pi i \Omega\left(X^{0}+D\right)} \mathrm{d} \Omega
$$

The outcome of Equation (12) is the appearance of $f\left(X^{0}+D\right)=f\left(X^{1}\right)$ on planet A while the transporter device remains on planet Earth. This means that the inside of the device has been transported to planet A.

Consolidating Equations (5), (6) and (12) into single direct form while taking under consideration that the integration remains unchanged within the transporter device $\mathrm{J}_{\mathrm{E}}$ on planet Earth, we get, 


$$
f\left(X^{1}\right)=c^{2} \int_{R^{3}} \int_{S_{E}^{2}} f(V) \mathrm{e}^{2 \pi i \Omega\left(X^{1}-V\right)} \mathrm{d} V \mathrm{~d} \Omega=c^{2} \int_{S_{E}^{2}} \int_{R^{3}} f(V) \mathrm{e}^{2 \pi i \Omega\left(X^{1}-V\right)} \mathrm{d} \Omega \mathrm{d} V
$$

$X^{1}$ represents the selected coordinates on planet A and $f\left(X^{1}\right)$ the appearance of the object $f(X)$ on planet A. $f(V)$ represents the internal space including the object within the device $\mathrm{J}_{\mathrm{E}}$.

Equation (13) provides a way to overcome the needs for storage facilities to store the Fourier frequencies wave pattern of the object. This issue by itself is a major challenge that needs further investigation.

The expression within the exponential of Equation (13), i.e.

$$
X^{1}-V=\left(x_{1}-v_{x}, y_{1}-v_{y}, z_{1}-v_{z}\right)
$$

represents the difference between the coordinate $X^{1}$ selected to be on planet A and the coordinates $V$ within the device $\mathrm{J}_{\mathrm{E}}$ located on planet Earth. All it takes is to know the desire location on planet A (Equation (14)) without the needs to travel the distance $D$ connecting between the two planets.

In order to reconstruct $f(X)$ in the exact unify body/object as of the origin, the topology structure of the coordinates of $f(X)$ on planet A must be identical to the origin on planet Earth, otherwise the reconstruction would be inhomogeneous in the sense that $f(X)$ could be spread in various locations on planet $\mathrm{A}$.

The summation in Equation (13) is commutative, one may first sum the frequencies and then the sphere coordinates or vice versa.

It seems bizarre, unrealistic situation that the outcome of frequency modulation with the object $f(X)$ located inside the device $\mathrm{J}_{\mathrm{E}}$ on planet Earth may cause to the appearance of the object $f(X)$ on planet $\mathrm{A}$, located hundreds of light years from Earth. Imagine that the object is human being.

One of the major questions-does the appearance of the object $f(X)$ on planet A represents a copy of the object or the object disappears from the transporter device $\mathrm{J}_{\mathrm{E}}$ and reappears on planet $\mathrm{A}$. To our best opinion, the execution of the Fourier integral over the internal spherical points in the device $\mathrm{J}_{\mathrm{E}}$, transform the object into new space, the frequency space. The object ceases to exist within the device $\mathrm{J}_{\mathrm{E}}$. The object is now coded as frequencies wave pattern. The inverse Fourier transform over the frequency space where the object is being hided or placed would reconstruct the object. But now, we can reconstruct the object in a different location since the frequency space does not depend on spatial location, distance, speed, time, we are free to reconstruct the object wherever we wish.

\section{Going Back from Planet A to Earth}

The procedure of going back from planet A to Earth requires sending probes to check what is going on in planet A. If the condition permit visit, then all parts of the device $\mathrm{J}_{\mathrm{E}}$ should transfer to planet $\mathrm{A}$, then engineers shall be transmitted to assemble the device $\mathrm{J}_{\mathrm{E}}$ on planet $\mathrm{A}$.

\section{Replicators or Jump Drive-Equivalent Concept}

Assuming that $\mathcal{F}(\Omega)$ is known and stored for certain items such as food, me- 
chanical or biological parts. This means that an item can be replicated once in any location. In StarTrack Voyager for example, a replicator was a machine that the crew have used to replicate food, parts and sometimes a complete device. Yet, in case $\mathcal{F}(\Omega)$ is stored in real facilities then objects cannot be replicated over and over, every replication is unique. Therefore, if we wish to replicate food, that means we need to store frequency wave patterns for example of 1 ton of meat and each time we will produce say $1 \mathrm{~kg}$ of meat until transforming the entire frequency wave patterns into 1 ton of meat.

On the other hand, we may assume that $\mathcal{F}(\Omega)$ of real object ceases to exist within the spaced-time universe, it's being transferred into the frequency space, a space without gravity and time. We may consider this space as the "zero space". In this case replication may not be limited.

Once the frequency wave pattern $\mathcal{F}(\Omega)$ of a certain item is known, then, the replicator can replicate the item in any location. The replicator is just a machine for executing the Fourier Transform on real objects, forward or backward.

\section{Time and Potential Time Travel}

The paper focused on spatial coordinate system while assuming fixed time. Fixed time may not be corrected since all planets are revolving. This means that the selected spatial location on planet A must be sync with the local time on Earth.

Furthermore, our basic assumption is that the temporal changes in the object during the transporting process is neglectable, that of course depends on the nature of the object, e.g. for living body this assumption may not be adequate.

Let assume that local Earth time interval $\left[t_{0}, t_{1}\right]$ is added to Equations (5) and (12), where the Fourier transform shall include frequency variable $\omega$ to be associated with time. In case the temporal changes of the object during the period [ $t_{0}$, $t_{1}$ ] are neglectable then the level of frequencies needed to reconstruct the temporal object would be non, i.e., only DC which means that the average appearance of the object over the time interval $\left[t_{0}, t_{1}\right]$ is good enough to represent the temporal appearance of the object. Let,

$$
\mathcal{F}(\Omega, \omega)=c \int_{t_{0}}^{t_{1}} \mathrm{e}^{-2 \pi i \omega t} \int_{R^{3}} f(X, t) \mathrm{e}^{-2 \pi i \Omega X} \mathrm{~d} X \mathrm{~d} t
$$

be the $4 \mathrm{D}$ spatial-temporal Fourier transform of $f(X, t)$, where outside the special-temporal domain $S_{E}^{2} \times\left[t_{0}, t_{1}\right]$ the object is considered to be zero.

The inverse Fourier transform is,

$$
f(X, t)=c \int_{R^{4}} \mathcal{F}(\Omega, \omega) \mathrm{e}^{2 \pi i \Omega X+\omega t} \mathrm{~d} \Omega \mathrm{d} \omega
$$

Using the shifting property,

$$
\begin{aligned}
& f\left(X^{0}+D, t_{0}+t_{k}\right)=f\left(X^{1}, t_{q}\right) \\
& =c^{2} \int_{R^{4}} \int_{S_{E}^{2}} \int_{t_{0}}^{t_{1}} f(V, t) \mathrm{e}^{2 \pi i\left[\Omega\left(X^{1}-V\right)+\omega\left(t_{q}-t\right)\right]} \mathrm{d} t \mathrm{~d} V \mathrm{~d} \Omega \mathrm{d} \omega
\end{aligned}
$$

where $t_{q}$ is the reconstructed time and $t_{k}$ is the time shift which may shift the object in time either forward or backward. $f\left(X^{1}, t_{q}\right)$ represents the appearance 
of the object in the new location $X^{1}$ and in the new time $t_{q}$. Let assume that the time phase element $t_{k}$ is larger or smaller than the time period $\left[t_{0}, t_{1}\right]$ which result either in the appearance of the object in the future time i.e. $t_{q}>t_{1}$ or in the past time i.e. $t_{q}<t_{0}$. Theoretically, there should not be a problem, but is there a practical problem? To make things clear, the object will not get older or younger, but it may appear as it was during the period $\left[t_{0}, t_{1}\right]$ in the future or the past timeline.

Since the Fourier transform is a non-causal operator, we may conclude that the time outside the interval $\left[t_{0}, t_{1}\right]$ may not necessarily match the future or the past of the current timeline $\left[t_{0}, t_{1}\right]$, i.e. there is no guarantee for the uniqueness of the timeline of the events outside the local time domain $\left[t_{0}, t_{1}\right]$. In order to guarantee identical timeline, certain conditions should be included to the transformation process. This issue is out of the scope of the current paper, and it will be dealt in our next papers.

Although the inverse is over the entire frequency range, further investigation on the actual bandwidth per each real object or material is required as indicated in Section 9.1.e hereunder. Figure 1 emphasizes the general idea:

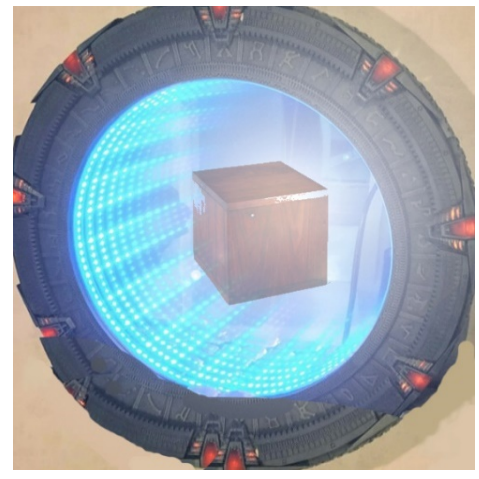

(a)

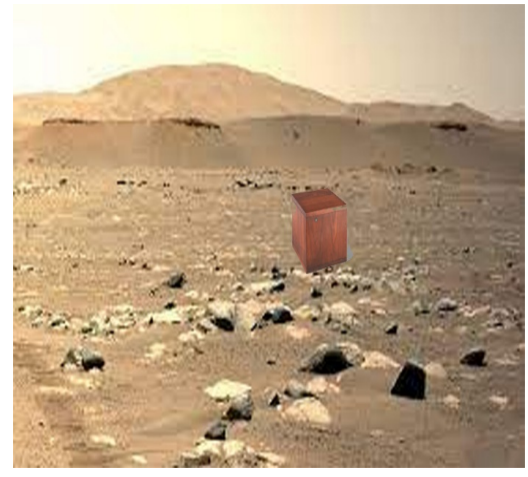

(b)

Figure 1. (a) The Transporter device JE located on planet Earth. The blue cells represent the kernel components of the $3 \mathrm{D} \times 4 \mathrm{D}$ spatial-temporal matrix $\mathrm{e}^{2 \pi\left[\Omega\left(X^{1}-V\right)+\omega\left(t_{q}-t\right)\right]}$ to be modulated with the BOX as part of the simultaneously execution of the forward-backward Fourier Transform for transporting the BOX to planet A.

$f\left(X^{0}+D, t_{0}+t_{k}\right)=f\left(X^{1}, t_{q}\right)=c^{2} \int_{R^{4}} \int_{S_{E}^{2}} \int_{t_{0}}^{t_{1}} f(V, t) \mathrm{e}^{2 \pi\left[\Omega\left(\Omega\left(X^{1}-V\right)+\omega\left(t_{q}-t\right)\right]\right.} \mathrm{d} t \mathrm{~d} V \mathrm{~d} \Omega \mathrm{d} \omega$; (b) The appearance of the BOX on planet A in the coordinates $X^{1}$ at synchronized planet A time $t_{q}$.

\section{Causality and Noncausality}

The key issue of the shift property is based on the noncausality characteristic of the Fourier Transform. Therefore, as long as, the recovery of the object is within the original spatial-temporal coordinate domain, the recovery would be part of the original spatial-temporal universe. However, if the spatial-temporal coordinates are out of the original domain, then there is no guarantee that the recovery would be in the same spatial-temporal Universe. The object may appear in a different universe representing different timeline. 


\section{Other Applications}

Acoustic or Voice and Video Holograms projection are one of the immediate applications of the suggested technique. The idea of holograms projection appears in Star war, where hologram message has been coded and appears as hologram projection without the need of a screen. For this purpose, we can store the Fourier transform of a $2 \mathrm{D}$ or $3 \mathrm{D}$ video message and transmit the message in different location. The result would be the appearance of the message as hologram projection without the need of a screen.

\section{Open Issues in the Path of Implementation}

There are so many variables and open issues in the path of implementing Equation (13). For example, creating $\mathcal{F}(\Omega)$ from real biological or mechanical parts? Would the reconstruction be identical to the origin? What would be the storage facilities? Part of the implementation issues are presented below.

a) Bandwidth and sampling intervals:

1) Spatial vs. Frequency sampling interval per each material e.g. living tissue, plastic, iron, copper, etc. The meaning of sampling intervals results in the design of the discrete $3 \mathrm{D} \times 3 \mathrm{D}$ kernel matrix,

2) $K\left(V, \Omega, X^{1}\right)=\mathrm{e}^{2 \pi i \Omega\left(X^{1}-V\right)}$ for fixed time.

Such that,

3) $\left\{\begin{array}{l}X_{n m k}^{1}=\left(x_{1}(n), y_{1}(m), z_{1}(k)\right) \\ V_{l p q}=\left(v_{x}(l), v_{y}(p), v_{z}(q)\right) \\ \Omega_{h g s}=\left(\Omega_{x}(h), \Omega_{y}(g), \Omega_{z}(s)\right)\end{array}\right.$

Including the process time duration,

4) $K\left(V, \Omega, X^{1}, \omega, t, t_{q}\right)=\mathrm{e}^{2 \pi i\left[\Omega\left(X^{1}-V\right)+\omega\left(t_{q}-t\right)\right]}$.

The 3Dx4D kernel matrix,

$$
\text { 5) }\left\{\begin{array}{l}
X_{n m k s}^{1}=\left(x_{1}(n), y_{1}(m), z_{1}(k), t_{q}(s)\right) \\
V_{l p i j}=\left(v_{x}(l), v_{y}(p), v_{z}(i), t(j)\right) \\
\Omega_{h g s b}=\left(\Omega_{x}(h), \Omega_{y}(g), \Omega_{z}(s), \omega(b)\right)
\end{array}\right.
$$

will be chosen in a way that the reconstruction of the object shall be $100 \%$ identical to the origin. The indices are all integers representing the sampling steps. For example, the index " $n$ " means $n a_{0}$ and the index " $h$ " means $h z_{0}$ where " $n$ " and " $h$ " are integers and $a_{0}$ and $z_{0}$ are real positive constants representing the spatial-frequency sampling intervals of the first component of the vectors $X^{1}$ and $\Omega$.

The events on planet Erath occur at timeline " $t$ " and the appearance of the object on planet A occurs at timeline $t_{q}$.

Furthermore, usually when applying DFT (FFT) there are two sets of coordinates, the forward and backward without geographical consideration, i.e. it does not matter if the computer is located in America or Africa, the execution of the DFT would be the same. However, in this case the backward coordinate location 
is not identical to the forward coordinate location as defined by the vectors $V$ (forward) and $X^{1}$ (backward). Therefore, the system in a.3 above introduces three sets of coordinates, two spatial vectors and one frequency vector while the system in a.5 incorporates additional three variables, Earth time, Destination time (on Planet A) and the frequency related to time.

Furthermore, the spatial sampling intervals of $V$ and $X^{1}$ may not be identical due to the differences in the environment between the two planets. Such differences may result from the changes in the local pressure, density, heat, gravitation, etc. Therefore, to overcome such differences, we may prepare within the transporter $\mathrm{J}_{\mathrm{E}}$ the destination environment enabling the object to easily adopt the destination environment while keeping identical sampling intervals for the two planets. Yet, this issue needs further investigation.

Signal processing teach us about Nyquist sampling rate, yet, since the objects are real parts such as food or even human, then, there is no place for error, the recovery of the object on planet $\mathrm{A}$, must be $100 \%$ identical to the origin.

6) Choosing the optimal frequency bandwidth $\left[\Omega_{0}, \Omega_{1}\right]$ per each material.

7) Does single bandwidth sufficient or there would be a need for several frequency bandwidths, i.e. $\left[\Omega_{0}, \Omega_{1}\right], \cdots,\left[\Omega_{k}, \Omega_{l}\right], \cdots,\left[\Omega_{m}, \Omega_{q}\right]$. This means that we may need for example: very short, short, near, mid, long, etc., wavelengths to be used per each material.

\section{b) Medium:}

What would be the optimal medium inside the device $\mathrm{J}_{\mathrm{E}}$ surrounding the object for improving the effectiveness of the frequency modulation with the object. For example, since substance of living tissue is composed mostly by water then it may be suggested that the surrounding environment would be water for improving the effectiveness of the frequency modulation.

\section{c) Energy:}

The optimal energy per frequency for best frequency modulation resulting in $100 \%$ recovery of the object.

\section{d) Storage:}

What sort of storage facilities shall be used to store the object frequencies wave pattern? As it seems, real object may not be coded into bits and bytes like we do in digital signal processing. So how and where should we store the real frequency wave pattern of meat for example?

e) The actual computation:

1) The implementation of the frequency modulation of the Fourier cubic kernel $K\left(V, \Omega, X^{1}\right)$ with the object within the transporter $\mathrm{J}_{\mathrm{E}}$.

2) Storage facilities of the frequency wave patterns, for example, food replicators.

3) The implementation of the Integrals.

These topics are opened for further research in the path to implement Jump drive, replicators, beam up transporters, etc. The detailed information about Fourier analysis can be found in many textbooks such as [12]. 


\section{Summary}

Jump drive, replicators, beam up transporters are feasible scientifically. Yet, it requires huge investment to develop such working device. There is a lot of space for future research since it involves real objects to be transported into the frequency space. For example, what sort of bandwidth and energy is needed to execute the Fourier transform forward and backward on a drop of water so that the drop of water shall disappear from the Jump drive device and reappear in the Jump drive device or in some other places?

In most applications, the matter absorbs the frequency energy, recalls for example X-ray tomography or in chemical markers identification such as of Raman technique. In this case, the frequencies energy should absorb the matter in the process of the Fourier integration, so that the matter will vanish from the spatial-temporal Universe and be converted into frequencies wave pattern.

One of the mysteries of the suggested method is the location of the object's frequencies wave pattern. In that sense, the object ceases to exist in the Universe. We may assume that the object frequencies wave pattern is located somewhere in the zero space, the space without time and gravity. Yet, this assumption needs further investigation. Avoiding the scenario for the needs to store the frequencies wave patterns, the execution of the forward-backward Fourier transform is done simultaneously.

Being optimistic, with the right design of the Fourier bandwidths and energy associated with each material to be transported, we may experience in our life the fiction devices of Star Trek and Star Gate.

\section{Acknowledgements}

I would like to give my special thanks to Shorok, Holok and Shalley for sharing knowledge with no boundaries while expending my pure consciousness.

Special thanks to Yehonatan Segman and David Solomon.

\section{Conflicts of Interest}

The author declares no conflicts of interest regarding the publication of this paper.

\section{References}

[1] Segman, Y.J. (2020) The Logical Perception of the Pure Consciousness. Science \& Philosophy, 8, 71-89.

[2] Segman, Y.J. (2020) The Logical Perception of the Origin of Matter and the Outside of the Universe. Science \& Philosophy, 8, 31-62.

[3] Alcubierre, M. (1994) The Warp Drive: Hyper-Fast Travel within General Relativity. Classical Quantum Gravity, 11, 73-77. https://doi.org/10.1088/0264-9381/11/5/001

[4] Segman, Y.J. (2021) Warp Drive with Positive Energy. Journal of High Energy Physics, Gravitation and Cosmology, 7, 906-913. https://doi.org/10.4236/jhepgc.2021.73051 
[5] Segman, Y.J., Rubinstein, J. and Zeevi, Y.Y. (1992) The Canonical Coordinate Method for Pattern Deformation: Theoretical and Computational Considerations. IEEE Transactions on Pattern Analysis and Machine Intelligence, 14, 1171-1183. https://doi.org/10.1109/34.177382

[6] Segman, Y.J. (1992) Fourier Cross Correlation and Invariance Transformations for an Optimal Recognition of Functions Deformed by Affine Groups. Journal of the Optical Society of America A, 9, 895-902. https://doi.org/10.1364/JOSAA.9.000895

[7] Segman, Y.J. and Schempp, W. (1993) Two Ways to Incorporate Scale in the Heisenberg Group with an Intertwining Operator. Journal of Mathematical Imaging and Vision, 3, 79-94. https://doi.org/10.1007/BF01248404

[8] Segman, Y.J. and Zeevi, Y.Y. (1993) Image Analysis by Wavelet-Type Transforms: Group Theoretic Approach. Journal of Mathematical Imaging and Vision, Special Issue on Wavelets, 3, 51-77. https://doi.org/10.1007/BF01248403

[9] Segman, Y.J. and Zeevi, Y.Y. (1992) The Spherical Wavelet Approach to Image Representation. Journal of Visual Communication \& Image Representation, 4, 263-270. https://doi.org/10.1006/jvci.1993.1024

[10] Segman, Y.J. and Schempp, W. (1992) On the Extension of the Heisenberg Group to Incorporate Multiscale Resolution. NATO Book on Wavelets and Their Applications, August 1992.

[11] Segman, Y.J. and Zeevi, Y.Y. (1992) A Wavelet-Type Approach to Image Analysis and Vision. NATO Book on Wavelets and Their Applications, August 1992.

[12] Alexander, W.E. and Williams, C.M. (2017) Digital Signal Processing: Principles, Algorithms and System Design. North Carolina State University, NC, USA. https://lib.ugent.be/catalog/ebk01:3710000001068972 